\title{
On Two-Ridge Structure in Two-Particle Azimuthal Correlations in Proton-Lead Collisions at Large Hadron Collider Energy
}

\author{
Fu-Hu Liu, Tian Tian, and Ya-Qin Gao \\ Institute of Theoretical Physics, Shanxi University, Taiyuan, Shanxi 030006, China \\ Correspondence should be addressed to Fu-Hu Liu; fuhuliu@163.com
}

Received 21 February 2014; Revised 23 April 2014; Accepted 29 April 2014; Published 20 May 2014

Academic Editor: Bao-Chun Li

Copyright ( $2014 \mathrm{Fu}-\mathrm{Hu}$ Liu et al. This is an open access article distributed under the Creative Commons Attribution License, which permits unrestricted use, distribution, and reproduction in any medium, provided the original work is properly cited. The publication of this article was funded by $\mathrm{SCOAP}^{3}$.

\begin{abstract}
Two-particle azimuthal correlations are studied in the framework of a multisource thermal model. Each source is assumed to produce many particles. Each particle pair measured in final state is considered to be produced at two emission points (subsources) in a single or two sources. The first emission point corresponds to the production of "trigger" particle and the second one corresponds to that of "associated" particle. There are oscillations and other interactions between the two emission points. In the rest frame of the "associated" particle's emission point, the oscillations and other interactions cause the momentum of the "trigger" particle to depart from the original value. The modelling results are in agreement with the experimental data of proton-lead $(p-\mathrm{Pb})$ collisions at $\sqrt{s_{N N}}=5.02 \mathrm{TeV}$, one of the Large Hadron Collider energies, measured by the ALICE and ATLAS Collaborations.
\end{abstract}

\section{Introduction}

Two-particle correlations are important experimental phenomenons in high energy collisions. These correlations contain azimuthal correlations, (pseudo)rapidity correlations, momentum correlations, and so on. As a type of long-range correlation, two-particle azimuthal correlations were studied in recent years. Particularly, at the eras of the Relativistic Heavy Ion Collider (RHIC) and the Large Hadron Collider (LHC), particle and nuclear physicists have been obtaining some interesting results on the azimuthal correlations in proton-proton $(p p)$, proton-nucleus, and nucleus-nucleus collisions. Some features are observed in the three types of collisions.

For example, the first studies of two-particle azimuthal correlation function in highest-multiplicity $p p$ collision at the LHC present an enhancement of particle pair production at relative azimuth $\Delta \phi \approx 0$, which results in a "ridge" structure at the "near-side" [1]. However, in peripheral proton-lead ( $p$ $\mathrm{Pb}$ ) collisions in which there are only a few nucleons to take part in the collisions and which are similar to $p p$ collision, a ridge structure is observed at the "away-side" $(\Delta \phi \approx \pi)$ [2]. The different results between $p p$ and peripheral $p-\mathrm{Pb}$ collisions are possibly caused by the infection of nuclear effects (spectator nucleons). In central $p$ - $\mathrm{Pb}$ collisions, a double ridge structure is observed, which is consistent with $\mathrm{Pb}-\mathrm{Pb}$ collisions [3].

To explain the near-side and away-side ridges, many physics mechanisms are proposed in literature, such as the parton saturation $[4,5]$, gluon saturation and color connections [6-11], parton-induced interactions [12-14], multiparton interactions [15], collective expansion of the final state [16], and collective effects arising in a high-density system [17-23]. If we regard a parton as an emission point and multiple emission points are assumed to form a source, the high energy collisions can be treated by a multisource interacting system. Thus, a multisource thermal model [2426] can be considered in the studies of near-side and awayside ridges in the two-particle azimuthal correlations. The effects listed here are not completely included in this study, but the collective effect is included.

In this paper, in the framework of the multisource thermal model [24-26], we consider two emission points (subsources) in a single or two sources. The two emission points are assumed to emit respectively the "trigger" particle and the "associated" particle in a particle pair. After considering oscillations and other interactions between the two emission points, the azimuthal correlations are studied. 


\section{The Model and Method}

In the multisource thermal model [24-26], we assume that many emission points (subsources) are formed in high energy collisions. These subsources can form a few sources which stay in local equilibrium sates. The Maxwell, Boltzmann, and other distributions can be used to describe spectrums of final-state particles in which productions of particle pairs are included. For a particle pair measured in final state, both the two emission points take part in the production process. The two emission points may be in a single or two sources. The first emission point corresponds to the production of "trigger" particle and the second one corresponds to the production of "associated" particle. There are oscillations between the two emission points, which results in the momentum of produced particle to depart from the original one. In the meantime, the multiple partons interactions [15], collective expansion of the final state [16], and other collective effects [17-23] can affect the momentum spectrums of final-state particles. Because the total physical extent of the interacting system is about 2 times the nuclear size, the average extent of a source is only a few $\mathrm{fm}$ in the case of considering two or three sources (temperatures).

Let the beam direction be the $o z$ axis, and let the transverse momentum direction of the "associated" particle be the positive direction of the $o x$ axis. A right-hand reference frame is established. In the rest frame of the second emission point, let $p_{x}$ and $p_{y}$ denote, respectively, the $x$-and $y$-components of the "trigger" particle momentum with oscillations and other interactions, and let $p_{x}^{\prime}$ and $p_{y}^{\prime}$ denote those without oscillations and other interactions. The simplest relations between $p_{x}$ and $p_{x}^{\prime}$, as well as $p_{y}$ and $p_{y}^{\prime}$ are linear:

$$
p_{x}=a_{x} p_{x}^{\prime}+b_{x} \sigma, \quad p_{y}=a_{y} p_{y}^{\prime}+b_{y} \sigma
$$

where $a_{x, y}$ and $b_{x, y}$ are parameters to characterize the strength of oscillations, and $\sigma$ is a parameter to characterize the distribution width of original momentum components. The default values of $a_{x, y}$ and $b_{x, y}$ are 1 and 0 , respectively.

From the original $p_{x, y}^{\prime}$ to the final-state $p_{x, y}$, a detailed consideration on the relativistic effect needs Lorentz transformation. Although there is no particular consideration on the relativistic effect in the above equations for the purpose of convenience, the present expression reflects approximately the mean result of the relativistic effect [24]. Meanwhile, the conservation laws are applicable in the interacting system. We would like to point out that in the above consideration both the "trigger" and "associated" particles are firstly assumed to emit isotropically from two subsources which have no oscillations and other interactions at this assumption state. Then, the momentum components are transformed from original $p_{x, y}^{\prime}$ to final-state $p_{x, y}$ due to the oscillations and other interactions between the two subsources.

As the first approximation, the original momentum components $p_{x, y}^{\prime}$ are assumed to obey Gaussian distribution with the width $\sigma$, which results in the transverse momentum, momentum, and nonrelativistic kinetic energy spectrums to be Rayleigh, Maxwell, and Boltzmann distributions, respectively. In the Monte Carlo calculation, let $r_{1,2,3,4}$ be random numbers distributed evenly in $(0,1]$. We have

$$
\begin{aligned}
& p_{x}^{\prime}=\sigma \sqrt{-2 \ln r_{1}} \cos \left(2 \pi r_{2}\right), \\
& p_{y}^{\prime}=\sigma \sqrt{-2 \ln r_{3}} \cos \left(2 \pi r_{4}\right),
\end{aligned}
$$

which distributes the azimuth of the particle randomly. The relative azimuth between the "trigger" and "associated" particles is

$$
\Delta \phi=\arctan \left[\frac{\sqrt{-2 \ln r_{3}} \cos \left(2 \pi r_{4}\right)}{a_{x} \sqrt{-2 \ln r_{1}} \cos \left(2 \pi r_{2}\right)+b_{x}}\right],
$$

due to $a_{y}=1$ and $b_{y}=0$ for the near-side and away-side ridge structures in general. A statistical calculation can give the normalization distribution $f(\Delta \phi)$ of $\Delta \phi$.

We would like to point out that (3) simply calculates the $\phi$ of the "trigger" particle using $\phi=\arctan \left(p_{y} / p_{x}\right)$. Although implied in the description of the coordinate system, it might be specified reiterated that $\Delta \phi=\phi_{\text {trig }}-\phi_{\text {assoc }}=\phi_{\text {trig }}$ due to $\phi_{\text {assoc }}=0$ (the transverse momentum direction of the "associated" particle being the positive direction of the $o x$ axis) can be represented using (3), where $\phi_{\text {trig }}$ and $\phi_{\text {assoc }}$ denote the azimuths of "trigger" and "associated" particles, respectively. Then, (3) uses (1) to obtain $\Delta \phi$. In addition, because we focus our attention on the relative magnitudes of $a_{x}$ and $a_{y}\left(b_{x}\right.$ and $\left.b_{y}\right)$, we may choose $a_{x}=1\left(a_{x}=0\right)$ or $a_{y}=1\left(b_{y}=0\right)$. Conventionally, we have $a_{y}=1$ and $b_{y}=0$.

The physics condition gives that $a_{x} \geq 1$. Generally, $a_{x}=1$ and $b_{x}=0$ describe the state without oscillations and other interactions. $a_{x}>1$ reflects an expansion of the subsource along $o x$ axis in the momentum space. $b_{x}>0$ and $b_{x}<0$ present respectively a near-displacement and an awaydisplacement of the first subsource to the second one along the $o x$ axis. The near-side and away-side phenomenons are partly determined by $b_{x}>0$ and $b_{x}<0$, respectively, and partly determined by $a_{x}>1$.

Before giving comparisons with experimental data, we need to introduce two representations which are used in the literature $[2,3,27]$. The first representation uses unidentified charged tracks as "trigger" particles and combines them with $\pi$ and $p$ as "associated" particles (denoted by $h-\pi$ and $h-p$, resp.) $[2,3,27]$. The correlation $C(\Delta \eta, \Delta \phi)$ is expressed in terms of the "associated" yield per "trigger" particle where both particles are from a given transverse momentum $\left(p_{T}\right)$ interval and pseudorapidity $(\eta)$ region:

$$
C(\Delta \eta, \Delta \phi)=\frac{1}{N_{\text {trig }}} \frac{d^{2} N_{\text {assoc }}}{d \Delta \eta d \Delta \phi}=\frac{S(\Delta \eta, \Delta \phi)}{B(\Delta \eta, \Delta \phi)},
$$

where $N_{\text {trig }}$ and $N_{\text {assoc }}$ are the numbers of "trigger" and "associated" particles, respectively, and $S(\Delta \eta, \Delta \phi)$ and $B(\Delta \eta, \Delta \phi)$ are the signal and background distributions [3,27] and constructed from the same event and "mixed events," respectively 
TABLE 1: Values of parameters and $\chi^{2} /$ dof corresponding to the curves in Figures 1-3.

\begin{tabular}{|c|c|c|c|c|c|c|}
\hline Figures & Type & $a_{x}$ & $b_{x}$ & $c_{1}\left(c_{2}\right)$ & $b_{0}$ & $\chi^{2} /$ dof \\
\hline Figure 1(a) & $0-20 \%, h-\pi$ & $1.045 \pm 0.002$ & $-0.020 \pm 0.002$ & $1.197 \pm 0.012$ & - & 0.872 \\
\hline Figure 1(b) & $(0-20 \%)-(60-100 \%), h-\pi$ & $1.040 \pm 0.002$ & $-0.005 \pm 0.001$ & $0.915 \pm 0.010$ & - & 1.129 \\
\hline Figure 1(c) & $(0-20 \%)-(60-100 \%), h-p$ & $1.030 \pm 0.002$ & $-0.003 \pm 0.001$ & $0.208 \pm 0.002$ & - & 1.105 \\
\hline Figure 2(a) & $0.3<p_{T}^{a}<0.5 \mathrm{GeV} / \mathrm{c}, \mathrm{C}$ & $1.040 \pm 0.002$ & $-0.024 \pm 0.002$ & $16.498 \pm 0.172$ & $-2.528 \pm 0.026$ & 1.051 \\
\hline Figure 2(b) & $0.5<p_{T}^{a}<1 \mathrm{GeV} / \mathrm{c}, \mathrm{C}$ & $1.040 \pm 0.002$ & $-0.020 \pm 0.002$ & $25.146 \pm 0.310$ & $-3.840 \pm 0.040$ & 0.595 \\
\hline Figure 2(c) & $1<p_{T}^{a}<2 \mathrm{GeV} / \mathrm{c}, \mathrm{C}$ & $1.040 \pm 0.002$ & $-0.019 \pm 0.002$ & $40.516 \pm 0.422$ & $-6.200 \pm 0.065$ & 0.470 \\
\hline Figure 2(d) & $2<p_{T}^{a}<3 \mathrm{GeV} / \mathrm{c}, \mathrm{C}$ & $1.040 \pm 0.002$ & $-0.020 \pm 0.002$ & $60.233 \pm 0.650$ & $-9.230 \pm 0.096$ & 0.282 \\
\hline Figure 2(e) & $3<p_{T}^{a}<4 \mathrm{GeV} / \mathrm{c}, \mathrm{C}$ & $1.040 \pm 0.002$ & $-0.025 \pm 0.003$ & $66.202 \pm 0.680$ & $-10.100 \pm 0.105$ & 0.356 \\
\hline Figure 2(f) & $4<p_{T}^{a}<5 \mathrm{GeV} / \mathrm{c}, C$ & $1.040 \pm 0.002$ & $-0.029 \pm 0.003$ & $69.403 \pm 0.710$ & $-10.600 \pm 0.110$ & 0.819 \\
\hline Figure 2(a) & $0.3<p_{T}^{a}<0.5 \mathrm{GeV} / \mathrm{c}, P$ & $1.010 \pm 0.001$ & $-0.045 \pm 0.005$ & $7.540 \pm 0.080$ & $-1.145 \pm 0.012$ & 0.755 \\
\hline Figure 2(b) & $0.5<p_{T}^{a}<1 \mathrm{GeV} / \mathrm{c}, P$ & $1.010 \pm 0.001$ & $-0.035 \pm 0.004$ & $14.363 \pm 0.145$ & $-2.215 \pm 0.024$ & 1.322 \\
\hline Figure 2(c) & $1<p_{T}^{a}<2 \mathrm{GeV} / \mathrm{c}, P$ & $1.012 \pm 0.001$ & $-0.030 \pm 0.003$ & $27.830 \pm 0.303$ & $-4.315 \pm 0.045$ & 1.101 \\
\hline Figure 2(d) & $2<p_{T}^{a}<3 \mathrm{GeV} / c, P$ & $1.013 \pm 0.001$ & $-0.030 \pm 0.003$ & $45.741 \pm 0.470$ & $-7.100 \pm 0.072$ & 0.575 \\
\hline Figure 2(e) & $3<p_{T}^{a}<4 \mathrm{GeV} / \mathrm{c}, P$ & $1.013 \pm 0.001$ & $-0.030 \pm 0.003$ & $60.354 \pm 0.630$ & $-9.360 \pm 0.095$ & 1.634 \\
\hline Figure 2(f) & $4<p_{T}^{a}<5 \mathrm{GeV} / \mathrm{c}, P$ & $1.013 \pm 0.001$ & $-0.025 \pm 0.003$ & $86.098 \pm 0.900$ & $-13.390 \pm 0.135$ & 1.903 \\
\hline Figure 3(a) & $0.3<p_{T}^{a}<0.5 \mathrm{GeV} / \mathrm{c}, C-P$ & $1.040 \pm 0.002$ & $-0.006 \pm 0.001$ & $12.533 \pm 0.122$ & $-1.950 \pm 0.020$ & 1.808 \\
\hline Figure 3(b) & $0.5<p_{T}^{a}<1 \mathrm{GeV} / \mathrm{c}, C-P$ & $1.040 \pm 0.002$ & $0.000 \pm 0.000$ & $21.923 \pm 0.220$ & $-3.400 \pm 0.037$ & 0.938 \\
\hline Figure 3(c) & $1<p_{T}^{a}<2 \mathrm{GeV} / \mathrm{c}, C-P$ & $1.040 \pm 0.002$ & $0.000 \pm 0.000$ & $32.036 \pm 0.332$ & $-4.950 \pm 0.051$ & 0.912 \\
\hline Figure 3(d) & $2<p_{T}^{a}<3 \mathrm{GeV} / \mathrm{c}, C-P$ & $1.040 \pm 0.002$ & $0.002 \pm 0.001$ & $42.163 \pm 0.405$ & $-6.520 \pm 0.066$ & 0.353 \\
\hline Figure 3(e) & $3<p_{T}^{a}<4 \mathrm{GeV} / \mathrm{c}, C-P$ & $1.040 \pm 0.002$ & $0.004 \pm 0.001$ & $50.203 \pm 0.527$ & $-7.790 \pm 0.080$ & 1.074 \\
\hline Figure 3(f) & $4<p_{T}^{a}<5 \mathrm{GeV} / \mathrm{c}, C-P$ & $1.040 \pm 0.002$ & $0.002 \pm 0.001$ & $40.485 \pm 0.500$ & $-6.280 \pm 0.065$ & 1.278 \\
\hline
\end{tabular}

$[2,28]$. If we integrate over $\Delta \eta$ in the above equation or if $\Delta \eta$ is a small value in general, we have

$$
C(\Delta \phi)=\frac{1}{N_{\text {trig }}} \frac{d N_{\mathrm{assoc}}}{d \Delta \phi}=\frac{S(\Delta \phi)}{B(\Delta \phi)} .
$$

Because the background $B(\Delta \phi)$ constructed from the isotropic "mixed events" in our model is a constant, we have

$$
C(\Delta \phi)=c_{1} S(\Delta \phi)=c_{1} f(\Delta \phi),
$$

where $c_{1}$ is the normalization constant and the signal $S(\Delta \phi) \equiv$ $f(\Delta \phi)$.

The second representation uses the "per-trigger yield" $Y(\Delta \phi)$ to measure the average number of particles correlated with each "trigger" particle, folded into $[0, \pi][1,29-31]$ and integrated over $\Delta \eta$ in $C(\Delta \eta, \Delta \phi), S(\Delta \eta, \Delta \phi)$, and $B(\Delta \eta, \Delta \phi)$ :

$$
Y(\Delta \phi)=\frac{\int B(\Delta \phi) d \Delta \phi}{\pi N_{a}} \cdot C(\Delta \phi)-b_{\mathrm{ZYAM}},
$$

where $N_{a}$ denotes the number of efficiency-weighted "trigger" particles and $b_{\mathrm{ZYAM}}$ represents the pedestal arising from uncorrelated pairs $[3,27]$. By using a zero-yield-atminimum (ZYAM) method $[29,32]$, the parameter $b_{\mathrm{ZYAM}}$ can be determined in experiments [3, 27, 29, 32]. Because $C(\Delta \phi)=c_{1} f(\Delta \phi)$, we have

$$
Y(\Delta \phi)=c_{2} f(\Delta \phi)+b_{0},
$$

where $b_{0}$ is a shift parameter which can be obtained by fitting experimental data and $c_{2}$ is the normalization constant.
To perform the calculation, we probe a set of $a_{x}$ and $b_{x}$ and use (3) to give a lots of $\Delta \phi$ by using many random numbers. Then, the normalized distribution $f(\Delta \phi)$ which is normalized to 1 can be obtained. Introducing $f(\Delta \phi)$ into (6) (or (8)) which is normalized to the experimental cross section or yield, the parameter $c_{1}$ (or the parameters $c_{2}$ and $b_{0}$ ) can be sounded out. By changing the parameters step by step, many repeating calculations can determine the best parameters and their uncertainties. In the real calculation, we can use the idea of the least-square fitting method. The minimum $\chi^{2}$ corresponds to the best parameter values, and the acceptable $\chi^{2}$ determines the uncertainties of the parameters.

\section{Comparison with Experimental Data and Discussions}

Figure 1 presents the correlations versus $\Delta \phi$ in transverse momentum interval $1.5<p_{T}<2.0 \mathrm{GeV} / \mathrm{c}$ in $p-\mathrm{Pb}$ collisions at $\sqrt{s_{N N}}=5.02 \mathrm{TeV}$, one of the LHC energies. The circles represent the experimental data of the ALICE Collaboration $[3,27]$ and the curves are our results calculated by the multisource thermal model. Figures 1(a), 1(b), and 1(c) correspond to $h-\pi$ in centrality $0-20 \%, h-\pi$ in centrality (0-20\%)-(60-100\%), and $h-p$ in centrality (0$20 \%)-(60-100 \%)$, respectively. The values of free parameters $a_{x}$ and $b_{x}$, normalization constant $c_{1}$, and $\chi^{2}$ per degree of freedom $\left(\chi^{2} /\right.$ dof $)$ obtained by fitting the experimental data are listed in Table 1. In the Monte Carlo calculation, we have used the idea of least-square fitting method. Many tries on the calculation have been applied to get the minimum and acceptable $\chi^{2}$. Then, the best parameter values and their 


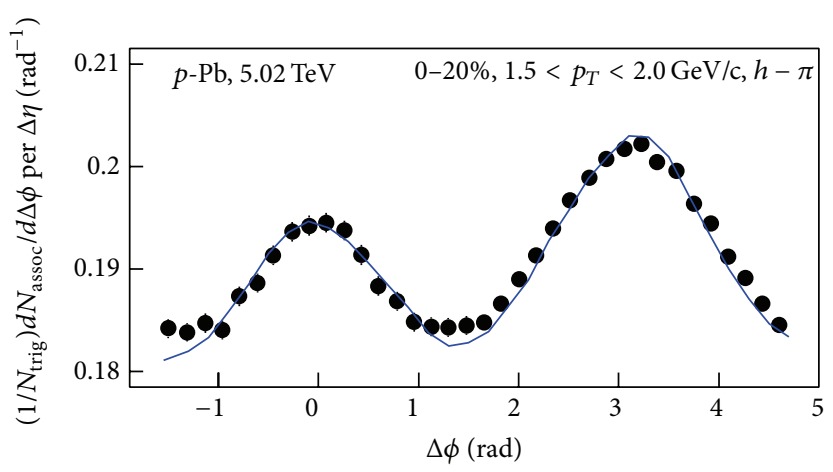

(a)

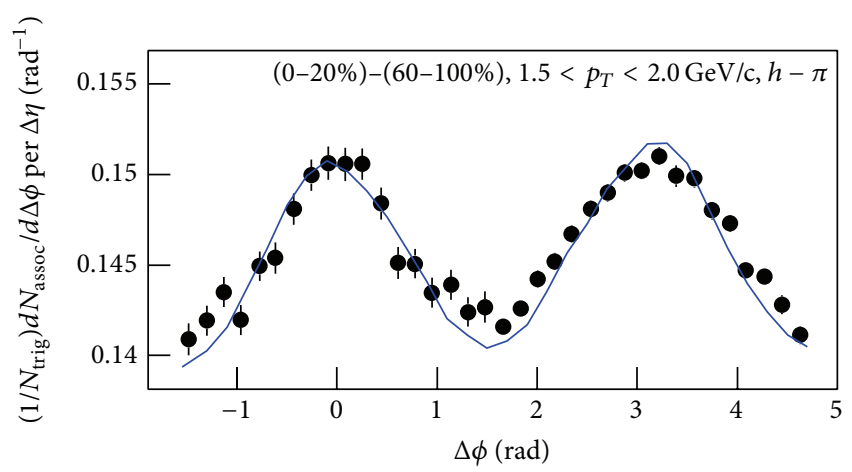

(b)

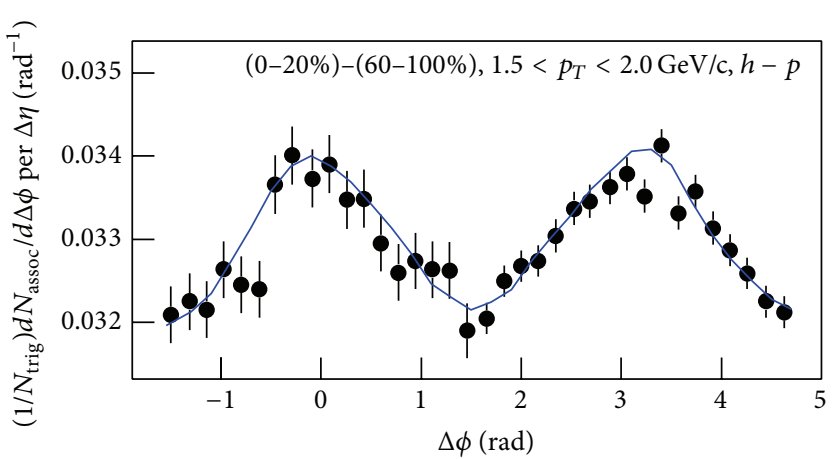

(c)

Figure 1: The correlations versus $\Delta \phi$ in transverse momentum interval $1.5<p_{T}<2.0 \mathrm{GeV} / \mathrm{c}$ in $p$ - $\mathrm{Pb}$ collisions at $\sqrt{s_{N N}}=5.02 \mathrm{TeV}$. The circles represent the experimental data of the ALICE Collaboration $[3,27]$ and the curves are our results calculated by the multisource thermal model. Figures $1(\mathrm{a}), 1(\mathrm{~b})$, and 1 (c) correspond to $h-\pi$ in centrality $0-20 \%, h-\pi$ in centrality $(0-20 \%)-(60-100 \%)$, and $h-p$ in centrality (0-20\%)-(60-100\%), respectively.

uncertainties can be determined. From the table, one can see that the model describes well the experimental data of the ALICE Collaboration. The subsource has an expansion and an away-displacement along the $o x$ axis.

Figure 2 shows $Y(\Delta \phi)-|\Delta \phi|$ relations in $p$ - $\mathrm{Pb}$ collisions at $\sqrt{s_{N N}}=5.02 \mathrm{TeV}$. The closed and open circles represent, respectively, the central (denoted " $C$ " in the panel) and peripheral collisions (denoted " $P$ " in the panel) measured by the ATLAS Collaboration [2], and the curves are our results calculated by the model. From Figures 2(a) to 2(f), different transverse momentum intervals for the "trigger" particle $\left(p_{T}^{a}\right)$ and for the "associated" particle $\left(p_{T}^{b}\right)$ are shown in the panels. The values of related parameters $a_{x}, b_{x}, c_{2}$, and $b_{0}$ as well as $\chi^{2} /$ dof are listed in Table 1. Once more, the model describes well the experimental data of the ATLAS Collaboration. In most cases, the subsource in central collisions has a larger expansion and a smaller away-displacement along the $o x$ axis, while the subsource in peripheral collisions is opposite. These differences between the central and peripheral collisions are rendered by the number of participant nucleons.

The difference $(C-P)$ on $Y(\Delta \phi)-|\Delta \phi|$ relations between the central and peripheral $p-\mathrm{Pb}$ collisions at $\sqrt{s_{N N}}=$ $5.02 \mathrm{TeV}$ is shown in Figure 3. The circles represent the experimental data of the ATLAS Collaboration [2] and the curves are our results calculated by the model. The values of related parameters and $\chi^{2} /$ dof are given in Table 1 . We see that the model describes the the difference between the central and peripheral collisions. The values of $a_{x}(=1.04)$ and $b_{x}(\approx 0)$ obtained from Figure 3 are consistent with those obtained from Figures 1(b) and 1(c). Because there is no direct proportional relation between the parameter values and the correlation magnitude, we cannot obtain simply the parameter values for Figure 3 by the method of central minus peripheral collisions.

From the above comparisons we see that we have used the same method to describe the central, peripheral, and central-peripheral collisions. Different participant nucleon numbers (different spectator nucleon numbers) in central and peripheral collisions reflect different parameter values. As an integrative result, the oscillation and other interactions existing between the two subsources cause $a_{x}$ to be greater than 1 and $b_{x}$ to be less than 0 . Generally, $a_{x}$ in central collisions is greater than that in peripheral collisions, and $\left|b_{x}\right|$ in central collisions is less than that in peripheral collisions, due to the effect of participant nucleon number. By comparison with Figures 1(a) and 2, as the difference between the central and peripheral collisions, Figures 1(b), 1(c), and 3 do not contain new content but the effect of participant nucleon number.

In the comparisons, because our calculation is performed on a purely signal sample, and experimental data are usually presented by a admixture of signal and background samples, we need the normalized constants $c_{1}$ and $c_{2}$. Our results show that $c_{1}>1$ and $c_{2}>1$ for both the central and 


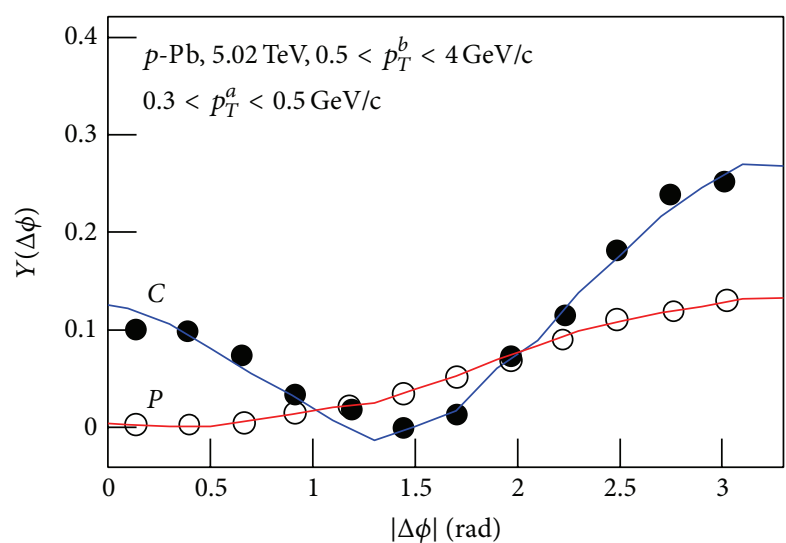

(a)

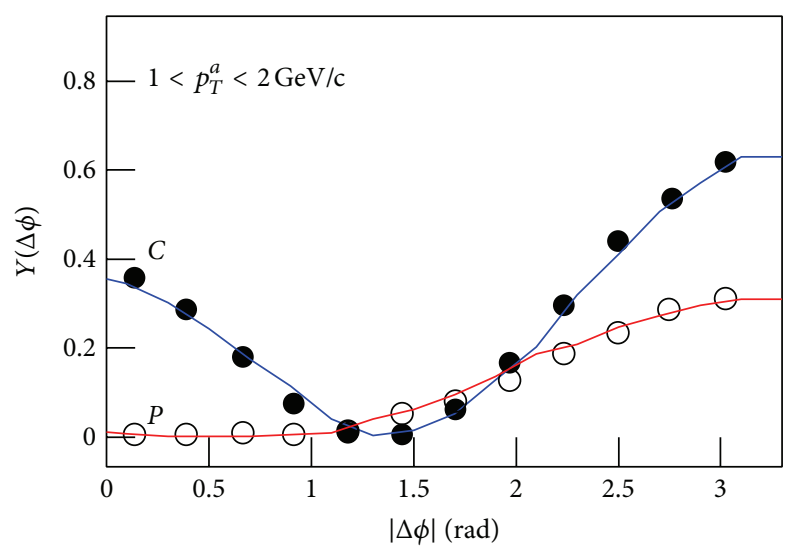

(c)

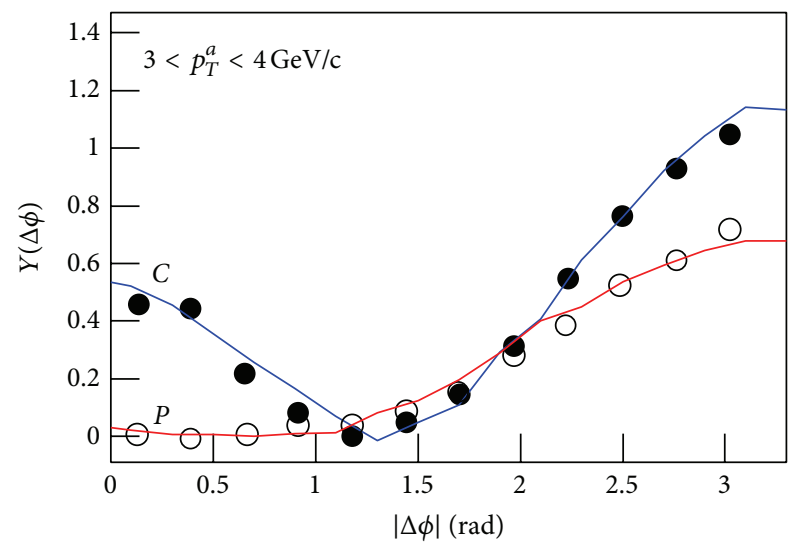

(e)

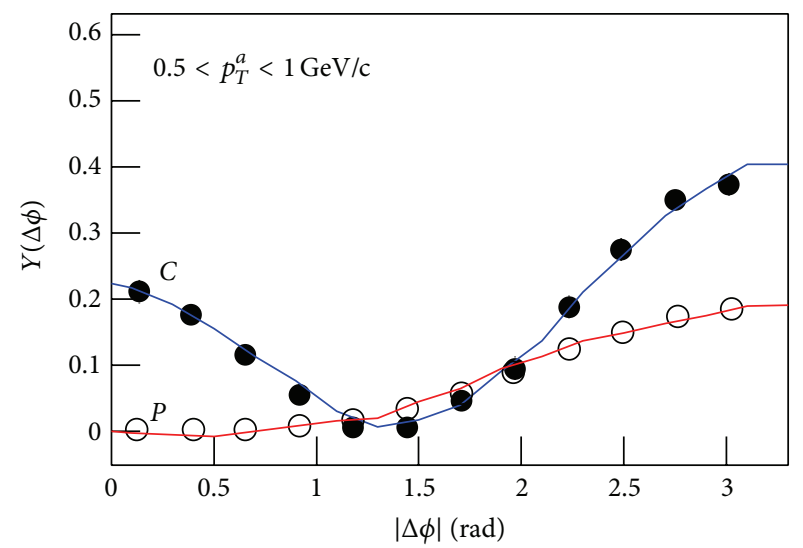

(b)

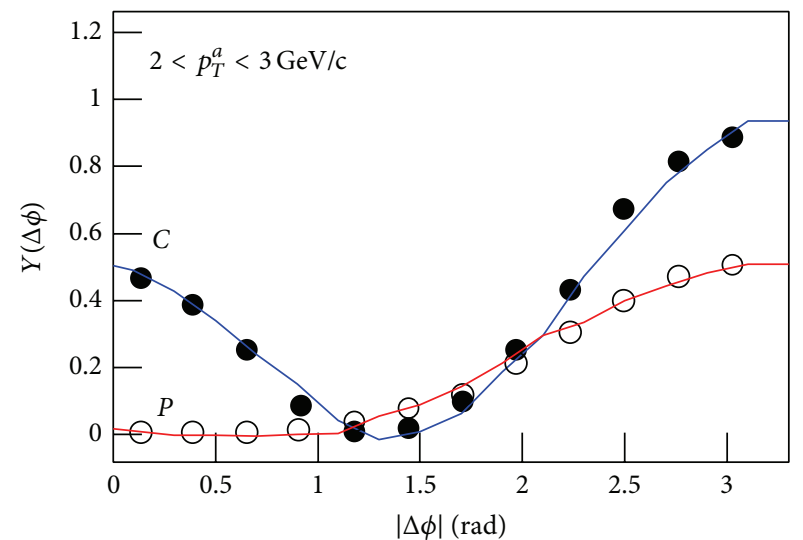

(d)

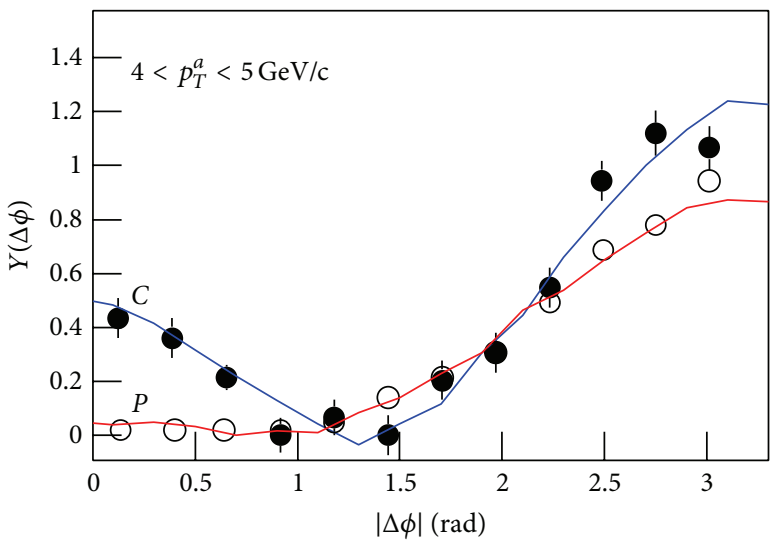

(f)

Figure 2: The relation $Y(\Delta \phi)-|\Delta \phi|$ in $p$-Pb collisions at $\sqrt{s_{N N}}=5.02 \mathrm{TeV}$. The closed and open circles represent, respectively, the central and peripheral collisions measured by the ATLAS Collaboration [2], and the curves are our results calculated by the multisource thermal model. From Figures 2(a) to 2(f), different transverse momentum intervals for the "trigger" particle $\left(p_{T}^{a}\right)$ and for the "associated" particle $\left(p_{T}^{b}\right)$ are shown in the panels.

peripheral collisions, which render that the magnitude of signal is greater than that of background. There are strong correlations between two particles in the production in $p-\mathrm{Pb}$ collisions at LHC energy.
Looking at the results in Table 1 , the source with oscillations becomes less displaced in peripheral collisions compared to central collisions. The standard deviation of the momentum components also becomes larger in peripheral 


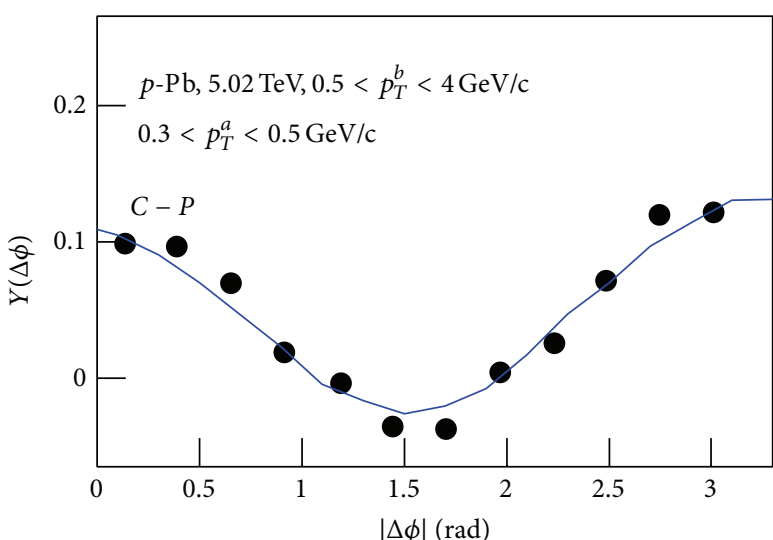

(a)

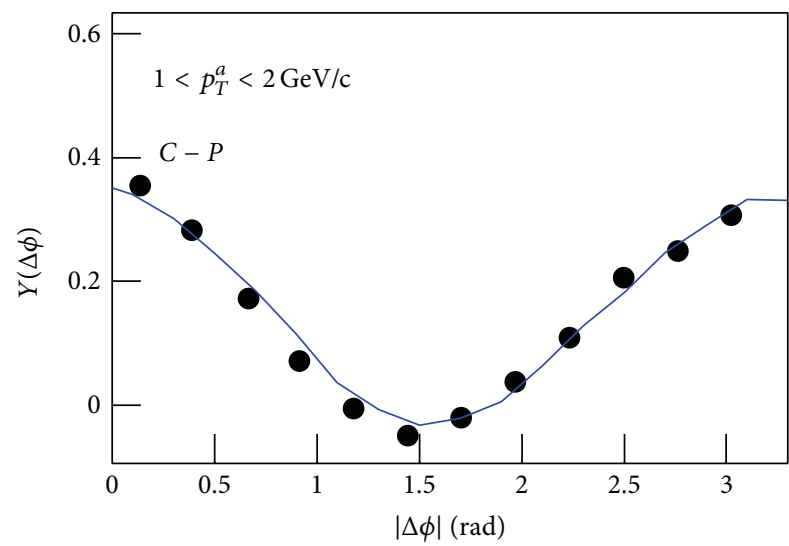

(c)

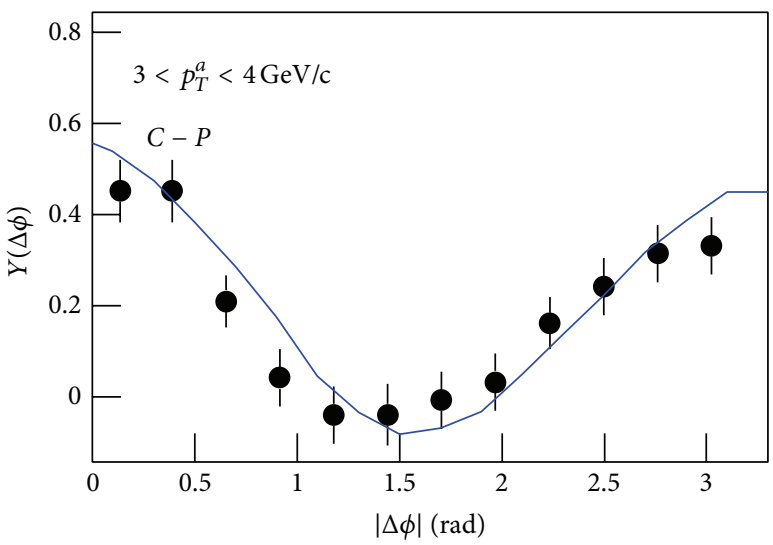

(e)

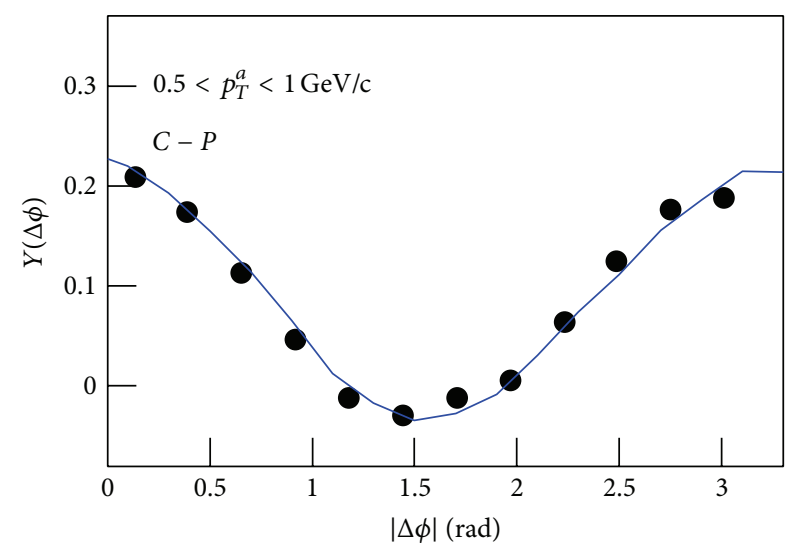

(b)

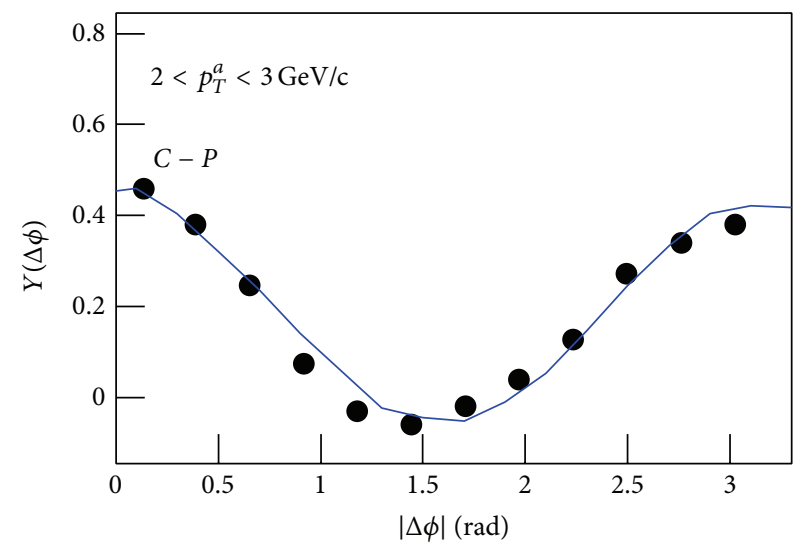

(d)

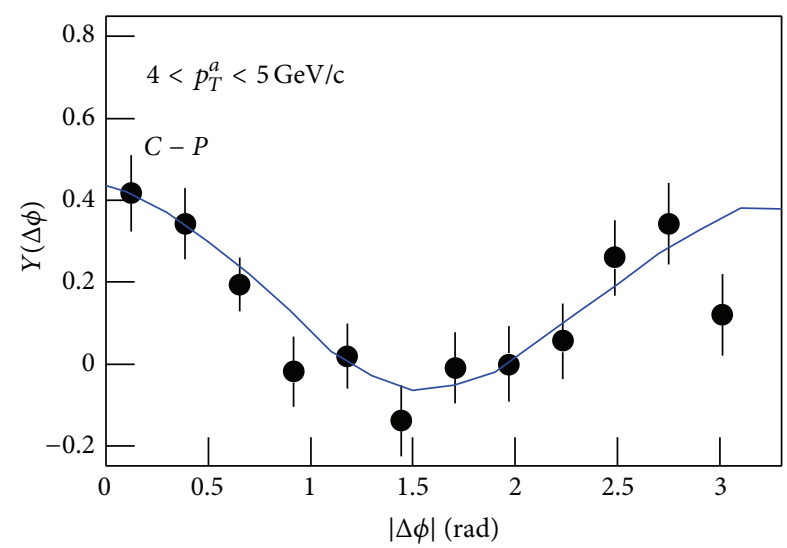

(f)

FIgURE 3: The same as Figure 2, but showing the difference between the central and peripheral $p$ - $\mathrm{Pb}$ collisions at $\sqrt{s_{N N}}=5.02 \mathrm{TeV}$.

collisions. These phenomenons may be caused by different numbers of participant nucleons in peripheral and central collisions.

In heavy ion collisions, the current consensus is that a primary component of the ridge effect is caused by fluctuations in the initial state geometry with a major contribution from "triangular flow," which generates a significant third Fourier coefficient contribution to the azimuthal correlations.
These may be the reasons which cause oscillations and other interactions between the two subsources. In fact, other reasons can cause similar results.

High energy collisions contain abundant contents such as multiplicity, transverse energy, entropy [33], phase transition [34], and flow effects [35]. There are strong relations between flow effects and azimuthal distributions and correlations. Particularly, the azimuthal distribution and correlations of 
produced particles, target fragments, and projectile fragments have important worth of studies. The present work can be referenced in further.

\section{Conclusions}

From the above discussions, we obtain following conclusions.

(a) The near-side and away-side ridge structures in two-particle azimuthal correlation produced in high energy collisions can be explained by the multisource thermal model. The modelling results are in agreement with the experimental data of $p-\mathrm{Pb}$ collisions at $\sqrt{s_{N N}}=5.02 \mathrm{GeV}$ measured by the ALICE and ATLAS Collaborations. This renders that our modelling assumption is correct. The two correlated particles are initially assumed to produce isotropically in two rest subsources. Then, the momentum components are transformed from original one to final-state one due to the oscillations and other interactions between the two subsources.

(b) Two emission points (subsources) are assumed and used to perform the calculation. One subsource corresponds to the production of "trigger" particle, and the other subsource corresponds to the production of "associated" particle. There are oscillations and other interactions between the two subsources, which results in the momentum of "trigger" particle, in the rest frame of "associated" particle's source, to depart from the original one.

(c) There are two main parameters, $a_{x}$ and $b_{x}$, in the modelling calculation on correlations. $a_{x}=1$ and $b_{x}=0$ describe the state without oscillations and other interactions. $a_{x}>1$ reflects an expansion of the subsource along the ox axis in the momentum space. $b_{x}>0$ and $b_{x}<0$ present, respectively, a near-displacement and an away-displacement of the "trigger" particle's subsource to the "associated" particle's subsource along the $o x$ axis. The magnitude of near-side and away-side ridges is partly determined by $b_{x}>0$ and $b_{x}<0$, respectively, and partly determined by $a_{x}>1$.

(d) In central and peripheral $p$ - Pb collisions at the LHC energy, our modelling results show that $a_{x}>1$ and $b_{x}<$ 0 . In most cases, the subsource in central collisions has a larger expansion and a smaller away-displacement along the $o x$ axis, while the subsource in peripheral collisions is opposite. The difference between the central and peripheral collisions shows a very small $b_{x}(\approx 0)$ which means a nearly zero displacement of the subsource. The values of $a_{x}$ and $b_{x}$ extracted from the central and difference $(C-P)$ data of the ALICE and ATLAS Collaborations are consistent with each other.

(e) The present model describes the central and peripheral $p$ - $\mathrm{Pb}$ collisions by using a uniform method. Although there are different participant nucleon numbers (or spectator nucleon numbers) between the central and peripheral collisions, the interacting mechanisms which include the oscillations and other interactions between the two subsources are the same except the intensity. The treatment on the difference $C-P$ does not introduce new content but the effect of participant nucleon number.

\section{Conflict of Interests}

The authors declare that there is no conflict of interests regarding the publication of this paper.

\section{Acknowledgments}

This work was partly finished at the State University of New York at Stony Brook, USA. One of the authors (Fu-Hu Liu) thanks Professor Dr. Roy A. Lacey and the members of the Nuclear Chemistry Group of Stony Brook University for their hospitality. The authors acknowledge the support of the National Natural Science Foundation of China (under Grants no. 10975095, no. 11247250, and no. 11005071), China National Fundamental Fund of Personnel Training (under Grant no. J1103210), the Open Research Subject of the Chinese Academy of Sciences Large-Scale Scientific Facility (under Grant no. 2060205), Shanxi Scholarship Council of China, and the Overseas Training Project for Teachers at Shanxi University.

\section{References}

[1] V. Khachatryan, A. M. Sirunyan, A. Tumasyan et al., "Observation of long-range, near-side angular correlations in protonproton collisions at the LHC," Journal of High Energy Physics, vol. 2010, no. 09, article 091, 2010.

[2] G. Aad, T. Abaiyan, B. Abbott, J. Abdallah et al., "Observation of associated near-side and away-side long-range correlations in $\sqrt{s_{N N}}=5.02 \mathrm{TeV}$ proton-lead collisions with the ATLAS detector," Physical Review Letters, vol. 110, no. 18, Article ID 182302, 18 pages, 2013.

[3] L. Milano, "Two-particle correlations in $\mathrm{p}-\mathrm{Pb}$ collisions at the LHC with ALICE," in Proceedings of the Strangeness in Quark Matter, Birmingham, United Kingdom, July 2013, http://arxiv.org/abs/1309.3457.

[4] A. Dumitru, K. Dusling, F. Gelis, J. Jalilian-Marian, T. Lappi, and R. Venugopalan, "The ridge in proton-proton collisions at the LHC," Physics Letters B, vol. 697, no. 1, pp. 21-25, 2011.

[5] K. Dusling and R. Venugopalan, "Azimuthal collimation of long range rapidity correlations by strong color fields in high multiplicity hadron-hadron collisions," Physical Review Letters, vol. 108, no. 26, Article ID 262001, 5 pages, 2012.

[6] B. Arbuzov, E. Boos, and V. Savrin, "CMS ridge effect at LHC as a manifestation of bremsstrahlung of gluons off quarks accelerated in a strong color field," The European Physical Journal C, vol. 71, no. 9, article 1730, 5 pages, 2011.

[7] K. Dusling and R. Venugopalan, "Evidence for BFKL and saturation dynamics from dihadron spectra at the LHC," Physical Review D, vol. 87, no. 5, Article ID 051502, 7 pages, 2013.

[8] K. Dusling and R. Venugopalan, "Explanation of systematics of CMS p+Pb high multiplicity dihadron data at $\sqrt{s_{N N}}=5.02 \mathrm{TeV}$," Physical Review D, vol. 87, no. 5, Article ID 054014, 12 pages, 2013.

[9] K. Dusling and R. Venugopalan, "Comparison of the color glass condensate to dihadron correlations in proton-proton and proton-nucleus collisions," Physical Review D, vol. 87, no. 9, Article ID 094034, 16 pages, 2013.

[10] Y. V. Kovchegov and D. E. Wertepny, "Long-range rapidity correlations in heavy-light ion collisions," Nuclear Physics A, vol. 906, pp. 50-83, 2013.

[11] A. Bzdak, B. Schenke, P. Tribedy, and R. Venugopalan, "Initial state geometry and the role of hydrodynamics in protonproton, proton-nucleus and deuteron-nucleus collisions," Physical Review C, vol. 87, no. 6, Article ID 064906, 10 pages, 2013. 
[12] C.-Y. Wong, "Momentum-kick model description of the ridge in $\Delta \varphi-\Delta \eta$ Correlations in $p p$ collisions at $7 \mathrm{TeV}$," Physical Review $C$, vol. 84, no. 2, Article ID 024901, 13 pages, 2011.

[13] M. Strikman, "Transverse nucleon structure and multiparton interactions," Acta Physica Polonica B, vol. 42, no. 12, pp. 26072630, 2011.

[14] S. Alderweireldt and P. Van Mechelen, "Obtaining the CMS ridge effect with multi-parton interactions," in Proceedings of the Workshop on Multi-Parton Interactions at the LHC, DESY, Hamburg, Germany, November 2011, http://arxiv.org/abs/1203.2048.

[15] M. G. Ryskin, A. D. Martin, and V. A. Khoze, "Probes of multiparticle production at the LHC," Journal of Physics G, vol. 38, no. 8, Article ID 085006, 11 pages, 2011.

[16] E. Avsar, C. Flensburg, Y. Hatta, J.-Y. Ollitrault, and T. Ueda, "Eccentricity and elliptic flow in proton-proton collisions from parton evolution," Physics Letters B, vol. 702, no. 5, pp. 394-397, 2011.

[17] K. Werner, I. Karpenko, and T. Pierog, “Ridge’ in proton-proton scattering at 7 TeV," Physical Review Letters, vol. 106, no. 12, Article ID 122004, 4 pages, 2011.

[18] W.-T. Deng, Z. Xu, and C. Greiner, "Elliptic and triangular flow and their correlation in ultrarelativistic high multiplicity proton-proton collisions at $14 \mathrm{TeV}$,' Physics Letters B, vol. 711, no. 3-4, pp. 301-306, 2012.

[19] E. Avsar, Y. Hatta, C. Flensburg, J.-Y. Ollitrault, and T. Ueda, "Eccentricity and elliptic flow in pp collisions at the LHC", Journal of Physics G, vol. 38, no. 12, Article ID 124053, 4 pages, 2011.

[20] P. Bożek, "Collective flow in $p-\mathrm{Pb}$ and $d-\mathrm{Pb}$ collisions at $\mathrm{TeV}$ energies," Physical Review C, vol. 85, no. 1, Article ID 014911, 9 pages, 2012.

[21] P. Bożek and W. Broniowski, "Correlations from hydrodynamic flow in pPb collisions," Physics Letters B, vol. 718, no. 4-5, pp. 1557-1561, 2013.

[22] P. Bożek and W. Broniowski, "Collective dynamics in highenergy proton-nucleus collisions," Physical Review C, vol. 88, no. 1, Article ID 014903, 13 pages, 2013.

[23] E. Shuryak and I. Zahed, "High-multiplicity pp and pA collisions: hydrodynamics at its edge," Physical Review C, vol. 88, no. 4, Article ID 044915, 13 pages, 2013.

[24] F.-H. Liu, "Particle production in Au-Au collisions at RHIC energies," Physics Letters B, vol. 583, no. 1-2, pp. 68-72, 2004.

[25] F.-H. Liu, "Dependence of charged particle pseudorapidity distributions on centrality and energy in $\mathrm{p}(\mathrm{d}) \mathrm{A}$ collisions at high energies," Physical Review C, vol. 78, no. 1, Article ID 014902, 6 pages, 2008.

[26] F.-H. Liu, "Unified description of multiplicity distributions of final-state particles produced in collisions at high energies," Nuclear Physics A, vol. 810, no. 1-4, pp. 159-172, 2008.

[27] B. Abelev, J. Adam, D. Adamova, A. M. Adare et al., "Long-range angular correlations of $\pi, \mathrm{K}$ and $\mathrm{p}$ in $\mathrm{p}-\mathrm{Pb}$ collisions at $\sqrt{s_{N N}}=$ 5.02 TeV," Physics Letters B, vol. 726, no. 1-3, pp. 164-177, 2013.

[28] A. Adare, S. Afanasiev, C. Aidala, N. N. Ajitanand et al., "Suppression of back-to-back hadron pairs at forward rapidity in $d+$ Au collisions at $\sqrt{s_{N N}}=200 \mathrm{GeV}$," Physical Review Letters, vol. 107, no. 17, Article ID 172301, 7 pages, 2011.

[29] A. Adare, S. Afanasiev, C. Aidala, and N. N. Ajitanand, "Dihadron azimuthal correlations in Au+Au collisions at $\sqrt{s_{N N}}$ $=200$ GeV," Physical Review C, vol. 78, no. 1, Article ID 014901, 42 pages, 2008.
[30] B. Alver, B. B. Back, M. D. Baker et al., "High transverse momentum triggered correlations over a large pseudorapidity acceptance in Au+Au collisions at $\sqrt{s_{N N}}=200 \mathrm{GeV}$," Physical Review Letters, vol. 104, no. 6, Article ID 062301, 4 pages, 2010.

[31] M. M. Aggarwal, Z. Ahammed, A. V. Alakhverdyants, I. Alekseev et al., "Azimuthal di-hadron correlations in $d+A u$ and $\mathrm{Au}+\mathrm{Au}$ collisions at $\sqrt{s_{N N}}=200 \mathrm{GeV}$ measured at the STAR detector," Physical Review C, vol. 82, no. 2, Article ID 024912, 14 pages, 2010.

[32] N. N. Ajitanand, J. M. Alexander, P. Chung et al., "Decomposition of harmonic and jet contributions to particle-pair correlations at ultrarelativistic energies," Physical Review C, vol. 72, no. 1, Article ID 011902, 5 pages, 2005.

[33] B.-C. Li and L.-L. Wang, "Entropy in scalar $O(N)$ model with a spontaneously broken symmetry," International Journal of Modern Physics A, vol. 24, no. 30, pp. 5725-5736, 2009.

[34] B. C. Li and M. Huang, "Strongly coupled matter near phase transition," Journal of Physics G, vol. 36, no. 6, Article ID 064062, 5 pages, 2009.

[35] B. C. Li, Y. Y. Fu, L. L. Wang, and F. H. Liu, "Dependence of elliptic flows on transverse momentum and number of participants in AuAu collisions at $200 \mathrm{GeV}$,' Journal of Physics $G$, vol. 40, no. 2, Article ID 025104, 9 pages, 2013. 

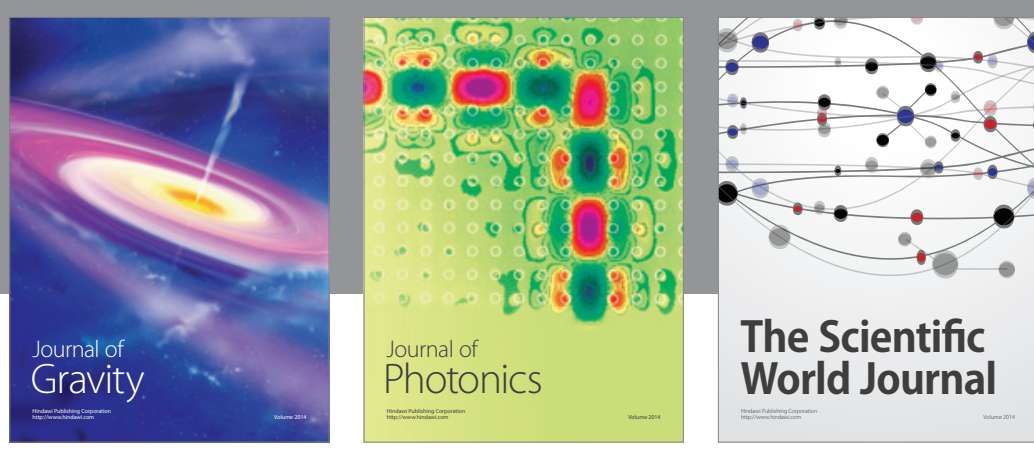

The Scientific World Journal
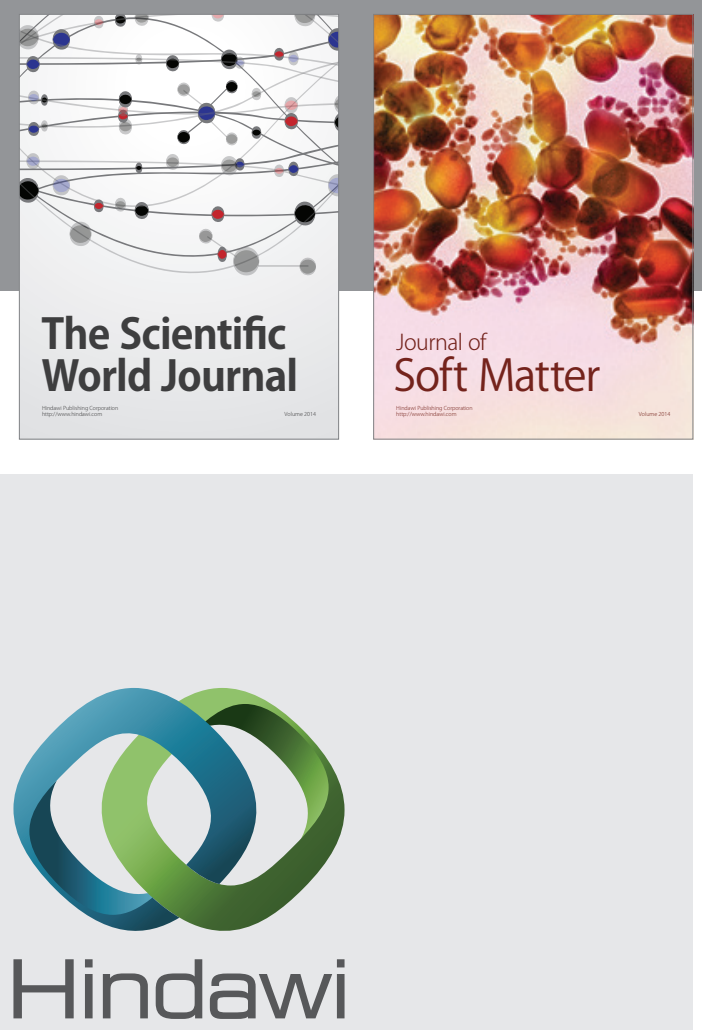

Submit your manuscripts at

http://www.hindawi.com

nternational Journal of

Statistical Mechanics
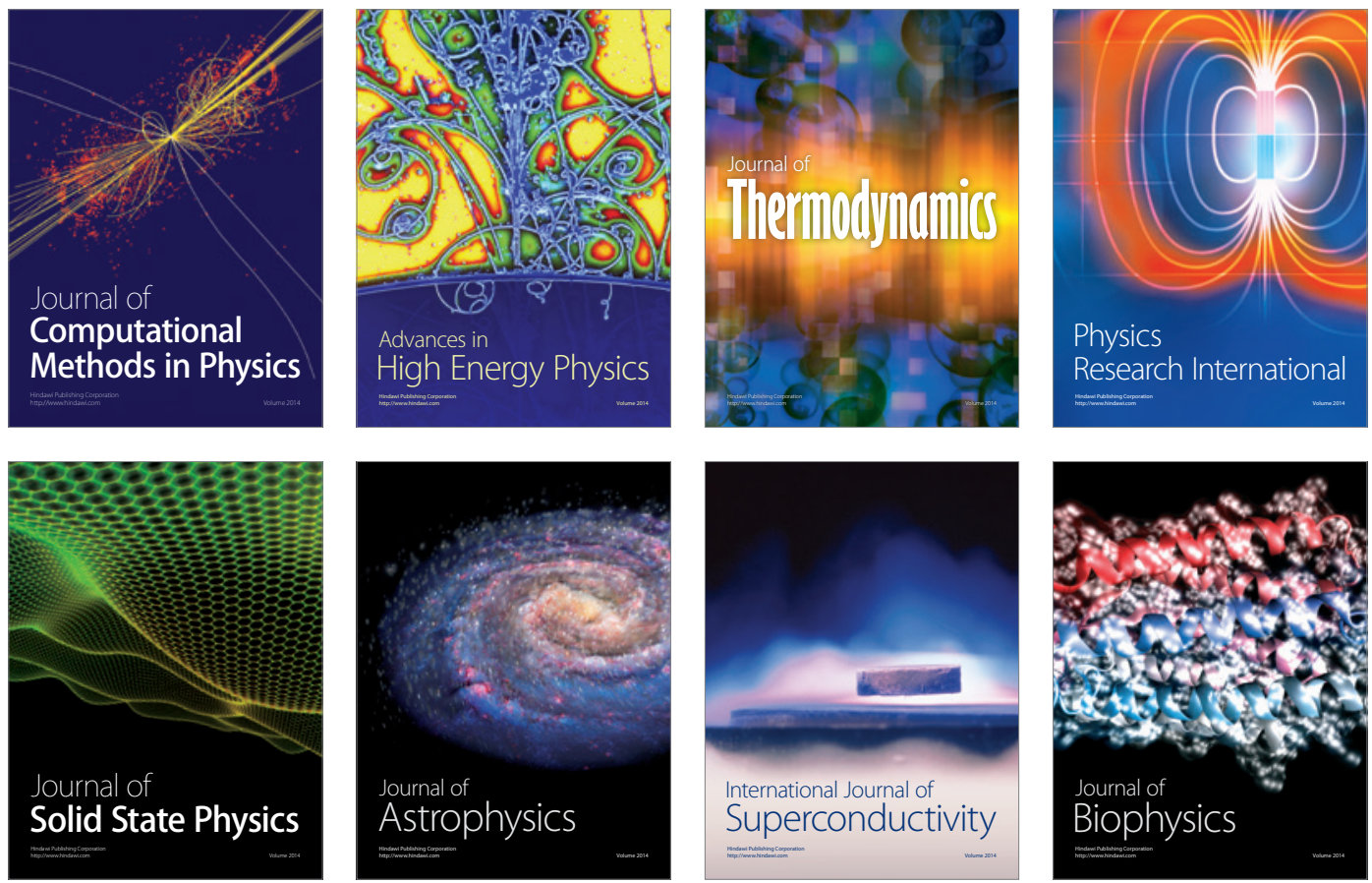
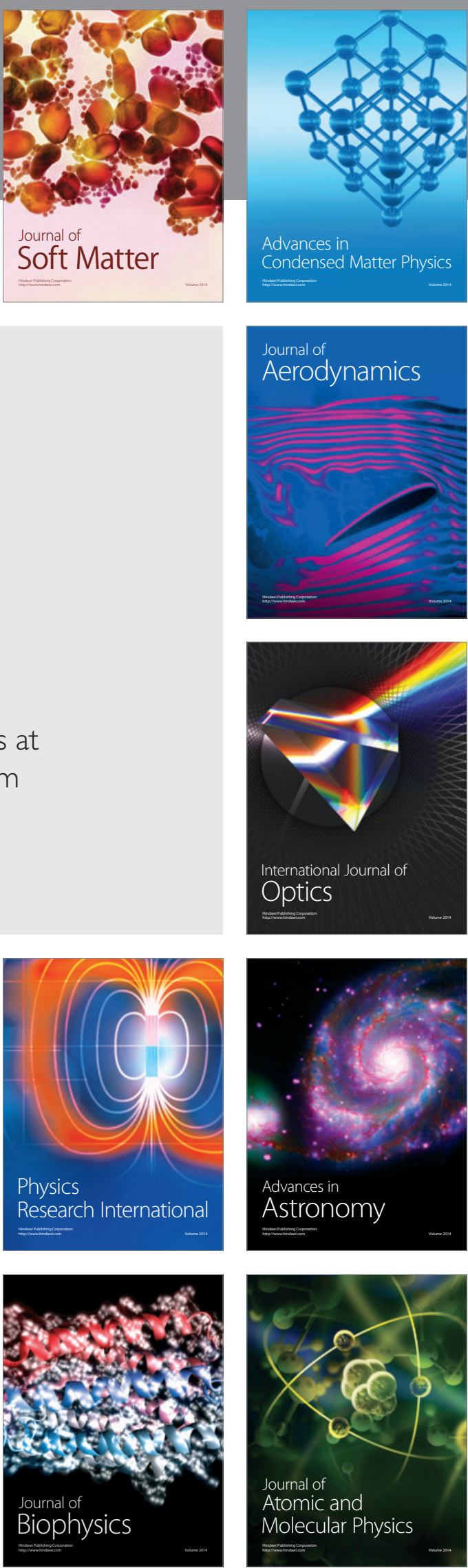